\title{
Addendum: Towards a UV completion of chameleons in string theory
}

\author{
Kurt Hinterbichler, ${ }^{a}$ Justin Khoury ${ }^{a}$ and Horatiu Nastase ${ }^{b}$ \\ ${ }^{a}$ Center for Particle Cosmology, Department of Physics and Astronomy, \\ University of Pennsylvania, 209 South 33rd Street, Philadelphia, PA 19104, U.S.A. \\ ${ }^{b}$ Instituto de Física Teórica, UNESP-Universidade Estadual Paulista, \\ R.Dr. Bento T. Ferraz 271, Bl. II, Sao Paulo 01140-0\%0, SP, Brazil \\ E-mail: kurthi@physics.upenn.edu, jkhoury@sas.upenn.edu, \\ nastase@ift.unesp.br
}

AdDENDUM TO: JHEP03(2011)061

KEYWORDS: Strings and branes phenomenology

Note that in KKLT, the scale appearing in $\rho$ is not the $4 \mathrm{~d}$ Planck scale, as written in eq. (4.1), but rather the $10 \mathrm{~d}$ string scale $M_{s}=\left(2 \pi \sqrt{\alpha^{\prime}}\right)^{-1}$, so $\sigma=M_{s}^{4} r^{4} / g_{s}=$ $M^{4} r^{4} /\left(2 \sqrt{\phi} g_{s}\right)$, where $M$ is the $10 \mathrm{~d}$ Planck scale. Since the experimental constraints require large extra dimensions $r$, this makes a big difference. $M$ is of course fixed, and $M_{\mathrm{Pl}}$ is given by experiment, so the difference between the two definitions can be absorbed by rescaling the prefactor $a$ appearing in the nonperturbative superpotential in eq. (4.3) by a large number, in which case we keep eq. (4.1) as it is.

We continued to refer to such a model as KKLT, though obtaining $a$ of order 1 as we assumed, as opposed to very small, is problematic for KKLT. Specifically, including this rescaling, KKLT have $a=2 \pi\left(M_{s} / M_{\mathrm{Pl}}\right)^{4}$ for Euclidean D3-brane instantons, and $a=$ $2 \pi / N_{c}\left(M_{s} / M_{\mathrm{Pl}}\right)^{4}$ for gluino condensation on D7-branes, modifying the expressions written below eq. (4.3). The experimental constraint on $R^{k}$ in eq. (5.11) implied a KK scale of $10^{11} \mathrm{GeV}$ in eq. (6.2). This in turn implies that the $10 \mathrm{~d}$ Planck scale is $M \simeq 10^{13} \mathrm{GeV}$, and $M_{s}^{4} / M_{\mathrm{Pl}}^{4} \sim 10^{-23}$, so we would need an $a$ which is $10^{23}$ times larger than natural. The same comment applies for the examples with $a<0$ from refs. $[65,66,67]$ cited in footnotes 4 and 5. It is possible to modify the model to work with $M$ rather than $M_{P l}$, but we will save this for future work. 